\title{
Selection of Wheat Ideotype Based on Multiple Traits using Genotype by Yield-Trait Approach
}

\author{
Muhammad Faheem $^{1 *}$, Khalil Ahmed Laghari ${ }^{1}$, Muhammad Khalil-Ur-Rehman ${ }^{2}$, Saima Mir Arain ${ }^{1}$ and \\ Mahboob Ali Sial ${ }^{1}$ \\ ${ }^{1}$ Division of Plant Breeding and Genetics, Nuclear Institute of Agriculture, Tando Jam, Sindh, Pakistan \\ ${ }^{2}$ Beijing Advanced Innovation Center for Tree Breeding by Molecular Design, Beijing Forest University, P. R. China \\ *For correspondence: mlofaheem@gmail.com \\ Received 06 January 2021; Accepted 16 February 2021; Published 10 May 2021
}

\begin{abstract}
In plant breeding, a novel genotype-by-yield trait (GYT) biplot approach was introduced to select superior genotypes based on multiple traits. The present study demonstrated the application of the GYT biplot model to evaluate the superior wheat advanced lines from a panel of 24 genotypes to select the ideotype for end users. Results show that the genotype-by-trait (GT) biplot covered $57 \%$ of the total variation of the data to reveal that grain yield was strongly associated with 1000 -grain weight and grain width. In contrast, the GYT biplot explained $90.2 \%$ of the total variation which was significantly much higher than GT biplot. According to tester vector view of GYT biplot almost all the yield trait combinations were associated with each other at different degree of association; whereas the genotypes present within the acute angles of tester vectors (yield trait combinations) had the trait profile contributed positively towards grain yield. The polygon biplot of GYT had eight sectors, out of which only three had the yield trait combinations. The eight genotypes were the polygon vertex among which the advanced line DF1906 of first sector was designated as the best genotype for spike length, number of spikelets per spike, grain weight per spike and number of grains per spike. Additionally, the DF1912 of second sector was early maturing coupled with high 1000-grain weight while DF1917 of third sector had short stature and gave the highest harvest index. The average tester coordination (ATC) biplot grouped 13 genotypes as superior and nine as inferior genotypes and recommended two advanced lines DF1912 and DF1917 as ideotype based on balanced traits profile. These findings strengthened the argument that the GYT biplot analysis is better than other selection indices and guaranteed the selection of superior genotypes and rejection of inferior ones based on multiple traits yield combinations. (C) 2021 Friends Science Publishers
\end{abstract}

Keywords: Wheat; Genotype by yield*trait biplot; Grain yield; Multiple traits

\section{Introduction}

Wheat (Triticum aestivum L.) is the most important food security crop in the world which provides basic calories and protein to $36 \%$ of the human population on earth ( $\mathrm{Li}$ et al. 2020). In Pakistan, wheat exclusively fulfills $60 \%$ of the caloric requirement of the population (Joshi et al. 2017). Despite the fact, the grain yield of wheat has increased many folds since the last few decades but the pace of improvement in yield (0.8-1.2\%) is not enough to feed 9-10 billion human population of the globe by 2050 (Tilman et al. 2011). To meet such a huge demand, wheat researchers are striving hard to accelerate the genetic gain for further improvement in the wheat grain yield and its quality.

Like other field crops, wheat also possesses a number of morphological and agronomic traits besides grain yield which must be considered during the evolution of high yielding cultivar. However, evaluation and selection of wheat genotypes based on multiple traits considering grain yield as the most important one is a tedious job due to the interaction of traits with the environment and also among themselves (Bernardo 2010). The scenario becomes more challenging when traits of interest are negatively correlated with each other (Yan et al. 2007). On the other hand, the wheat growers always expect and demand that new wheat cultivars should outclass the old cultivars specifically in grain yield coupled with improved agronomic traits and disease resistance (Merrick et al. 2020). Under such circumstances, selection based on multiple traits needs deep insight about genotype trait relationship and association among traits to combine optimum values of traits of interest in a single genotype. Yan et al. (2019) argued that the economic value of any trait in a cultivar depends upon the level of the main target trait i.e., yield. For instance, lodging resistance in wheat has the economic value only when it is coupled with high grain yield. Similarly, a genotype

To cite this paper: Faheem M, KA Laghari, M Khalil-Ur-Rehman, SM Arain, MA Sial (2021). Selection of wheat ideotype based on multiple traits using genotype by yield-trait approach. Intl J Agric Biol 25:1367-1374 
possessing high level of quality traits but with poor grain yield has low economic value of trait due to the fact that this genotype cannot be recommended as a cultivar. Hence, the main purpose of multiple trait selection is to pyramid the desirable traits with higher grain yield in a single genotype in such a way that all the traits escalate the economic value of one another.

Different conventional and molecular breeding approaches have been devised to tailor wheat genotype depicting a balance between grain yield with other agronomic traits not only under optimal environmental conditions but also under various biotic and abiotic stresses. Among conventional breeding approaches, tandem selection (Simmonds and Smartt 1999), independent culling (Godshalk et al. 1988), and index selection (Bos and Caligari 2007) are well known, effective, and common practiced tools to breed a cultivar based on multiple traits (Boureima and Abdoua 2019; Yan et al. 2019). But all these approaches rely on some truncation points or weightages which are at the disposal of researcher's choice, hence resulted in different selection outputs from the same dataset (Yan and Fregeau-Reid 2018). To remove such biasness, recently a novel genotype-by-yield trait (GYT) biplot approach was introduced in plant breeding to select superior genotypes based on multiple traits (Yan and Fregeau-Reid 2018). Basically, the GYT biplot is extension of genotype by trait (GT) biplot analysis which relies on combing different traits with yield (Merrick et al. 2020). The different GYT biplot views provide the accurate mean to display the ranking of genotypes according to the superiority of yield trait combinations and also the trait profile of each genotype to depicts its weaknesses and strength (Yan et al. 2019). After the pioneer study in oats (Yan and Fregeau-Reid 2018), this model was successfully practiced in other crops such as Hordeum vulgare (Karahan and Akgun 2020), Sesamum indicum (Boureima and Abdoua 2019) T. durum (Kendal 2019; Mohammadi 2019), and spring wheat (Merrick et al. 2020) to select the superior genotypes based on multiple traits. The same model (GYT biplot) was applied in this study to get better understanding about the associations of desirable traits in combination with key grain yield trait in elite wheat germplasm and subsequently to identify the superior genotypes based on multiple traits selection to speed up the genetic gain of wheat grain yield improvement in Pakistan.

\section{Materials and Methods}

\section{Plant material and experimental design}

The plant material included 23 wheat elite advanced lines along with a commercial check $c v$. NIA-Saarang was used in this study. These advance lines were developed by wheat breeding group of Nuclear Institute of Agriculture (NIA), Tando Jam, Pakistan. The check cultivar NIA-Saarang is a high yielding, rust resistant, and widely adopted wheat variety of NIA. The trials were conducted in the first week of November at experimental farm of NIA over two consecutive growing seasons (2018-19 and 2019-20). In 2018-19, alpha lattice experimental design with two replications was adopted to evaluate these advance lines against the commercial check while in 2019-20 the same genotypes were evaluated in randomized complete block design with three replications. The plot size of each genotype for both the trials was 9 square meter comprising six rows of 5 -meter length. To maintain the healthy growth of wheat plots, the recommended dose of fertilizers $\left(120 \mathrm{~kg} \mathrm{ha}^{-1}\right.$ nitrogen $60 \mathrm{~kg} \mathrm{ha}^{-1}$ potassium and $60 \mathrm{~kg} \mathrm{ha}^{-1}$ phosphorous) were applied. The crop cycle for each season was completed with four irrigations while other weed control standard practices were followed as established in the region.

For both the growing seasons, data regarding agronomic traits like days to heading (DH) and days to physiological maturity (DM) were recorded for each plot. At maturity, ten plants were randomly selected from the central rows of each plot of all the genotypes for each replication. Data regarding plant height $(\mathrm{PH})$ and yield components viz., spike length (SL), number of spikelets per spike (SPS), number of grains per spike (GS), grain weight per spike (GWS), and thousand kernel weight (TGW) were recorded from these plants. To determine the grain length (GL) and grain width (GW), the cumulative grain length and width of five grains were measured to compute the respective parameters of one grain. For biological yield, four central rows were harvested and weighed, subsequently, the same bundle was threshed to record grain yield per plot (GY) and expressed as tons per hectare. Harvest index (HI) was computed from biological yield and grain yield per plot to express in percentage.

\section{Statistical analysis}

The dataset was subjected to a novel approach of genotype by yield-trait biplot analysis following the protocol as described by Yan and Fregeau-Reid (2018). In brief, at the first step overall means and standard deviation for two years for all the parameters including grain yield were computed. This genotype trait data table was used to find the association among different traits and also for genotype by trait (GT) biplot analysis. Besides this, the GT data table was transformed to genotype by yield-trait (GYT) table by either multiply or dividing grain yield of each genotype with its respective parameter depending upon the breeding objectives. So, in the GYT table grain yield was divided by DH, DM, and PH with a notation of "/" as our objective of developing wheat advance lines were early maturing with short stature which could resist lodging. While all the other yield components (SL, SPS, GWS, TGW, GL, and GW) including harvest index was multiplied (*) with grain yield as larger means of these traits were more desirable. Eventually, before the final evaluation of genotypes the GYT table was standardized to remove the differences in 
the measuring units of yield trait combinations according to following equation:

$$
P_{i j}=\frac{T_{i j}-T_{J}}{S_{j}}
$$

Where Pij represents the standardized value of ith genotype for the trait or yield-trait combination $\mathrm{j}$ in the standardized table, Tij is the original value of genotype i for trait or yieldtrait combination $\mathrm{j}$ in the GT or GYT tables, $\mathrm{Tj}$ is the mean across genotypes for trait or yield-trait combination $\mathrm{j}$, and $\mathrm{Sj}$ is the standard deviation for trait or yield-trait combination $j$. This standardized dataset of GYT was then subjected to GYT biplot analysis and to calculate the mean superiority index value of each genotypes. The GT biplot and different views of GYT biplot were constructed by GGEbiplot software v. 8.2 following same software setting as suggested by Yan and Fregeau-Reid (2018).

\section{Results}

\section{Association among traits and trait profile based on genotype by trait (GT) biplot}

The GT biplot analysis was applied to the standardized data set of two growing seasons of 24 wheat genotypes of wheat to get deep insight about the relationship among the traits. The GT biplot presented in Fig. 1 was based on the principles (scaling $=1$, centering $=2$ and $\mathrm{SVP}=2$ ) laid down by Yan and Fregeau-Reid (2018) to represent the correlation among the traits and with the genotype which covered 57\% of the total variation of the data by plotting two main principal components $(\mathrm{PC} 1=36.4 \%$ and $\mathrm{PC} 2=20.6 \%)$. The GT biplot revealed that grain yield (GY) formed acute angle with thousand grain weight (TGW), hence strongly interlinked with one another, whereas, weak positive association was also found among GY, grain width (GW) harvest index (HI), grain weight per spike (GWS) and grain length (SL) (Fig. 1). Contrarily, all other traits either formed right angel or obtuse angles $\left(>90^{\circ}\right)$ with GY hence, depicted no or negative association with GY. The Pearson correlation of the same data set presented in Fig. 2 also confirmed the findings of GT biplot with some exceptions. For instance, correlation analysis suggests significant positive correlation between GY and HI while no significant association was found for other traits including TGW (Fig. 2). Similarly, another important yield component i.e., GWS formed acute angels with TGW, GL, PH, SL, GS, DM and SPS to represent strong association of these traits with GWS as well as among each other. In fact, the GT biplot formed a cluster of multiple traits viz., GWS, GL, PH, SL, GS, DM SPS and $\mathrm{DH}$ to depict the positive association of diverse strength among these traits depending upon the magnitude of angle between any of two given traits (Fig. 1). Additionally, all the members of this cluster had obtuse angle with $\mathrm{HI}$ to represent negative correlation with HI. The values of Pearson correlation graphically displayed in Fig. 2, also validated the same trend of association among traits as revealed by GT biplot.

Graphical display of trait profiles of genotypes is another unique feature of GT biplot which mainly depends upon the total variation explained by the biplot. The GT biplot shows that the genotype DF1912, DF1903, DF1917, and DF1915 produced better grain yield than check cultivar i.e., NIA-Saarang and other contesting genotypes and also showed better trait profiles for TGW and GW (Fig. 1). Similarly, DF1923 had the maximum HI as compared to rest of genotypes while other genotypes with good profile of HI were NIA-Saarang, DF1920, DF1918, and DF1916. The genotypes DF1910, DF1907, DF1908, DF1906, DF1905, DF1904, and DF1909 were found within the cluster of multiple traits viz., GWS, GL, PH, SL, GS, DM SPS and DH depicted good trait profile for these traits as these traits had positive association with each other but performed poor for $\mathrm{HI}$ and GW. The advanced lines DF1901, DF1911, DF1914 and DF1919 clustered away from all trait vectors under consideration, hence, had poor trait profile for the traits (Fig. 1).

\section{Association among traits based on grain yield by Genotype by yield-trait (GYT) biplot}

To select the best genotypes among the contesting advanced lines based on multiple traits, a novel approach of GYT biplot was applied considering grain yield as the most important economical trait. The GYT biplot analysis represented the $90.2 \%$ of the total variation by plotting first two principal components $(\mathrm{PC} 1=79.5 \%$ and $\mathrm{PC} 2=$ $10.7 \%$ ) in three exclusive biplot views named as the tester vector view (Fig. 3), the polygon view (Fig. 4), and the average tester coordination (ATC) view (Fig. 5). The ATC view of GYT biplot clearly demonstrated the two clusters of yield trait combinations, one having GY/PH, GY*HI, GY/DH, GY/DM and GY*GW while other consisted of GY*SL, GY*SPS, GY*GWS and GY*GS (Fig. 3). In first cluster, GY/PH and GY*HI had very strong correlation while GY/DH, GY/DM and GY*GW had same level of relationship among each other. In other cluster, GY*SL and GY*GWS exhibited strong relationship with GY*SPS and $G Y^{*} \mathrm{GS}$, respectively. However, wider angle between the members of these clusters presented weak relationship among yield trait combinations, specifically for GY/PH and GY*HI with the members of second group (Fig. 3). Two yield trait combinations i.e., GY*TGW and GY*GL highly correlated with each other were found in between these two clusters and showed the adequate level of relatedness for all the yield trait combinations.

\section{Selection of the best genotype based on multiple traits}

The polygon views also known as which is best for what biplot was constructed for all the yield trait combination to graphically present the trait profile of contesting genotypes 


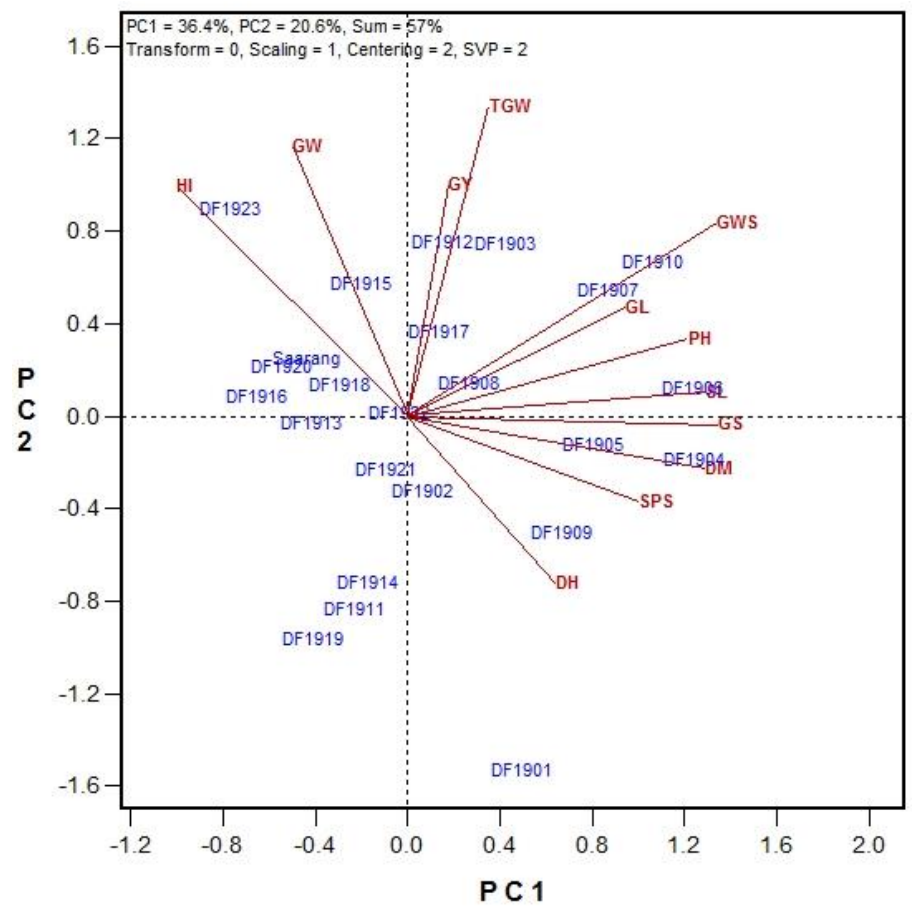

Fig. 1: The genotype by trait biplot based on standardized data of 23 wheat advanced lines and check 'NIA-Saarang' GY, grain yield; DH, days to heading; DM, days to physiological maturity; PH, plant height; SL, spike length; SPS; number of spikelets per spike; GS, number of grains per spike; GWS, grain weight per spike; TGW, 1000-grain weight; HI, harvest index

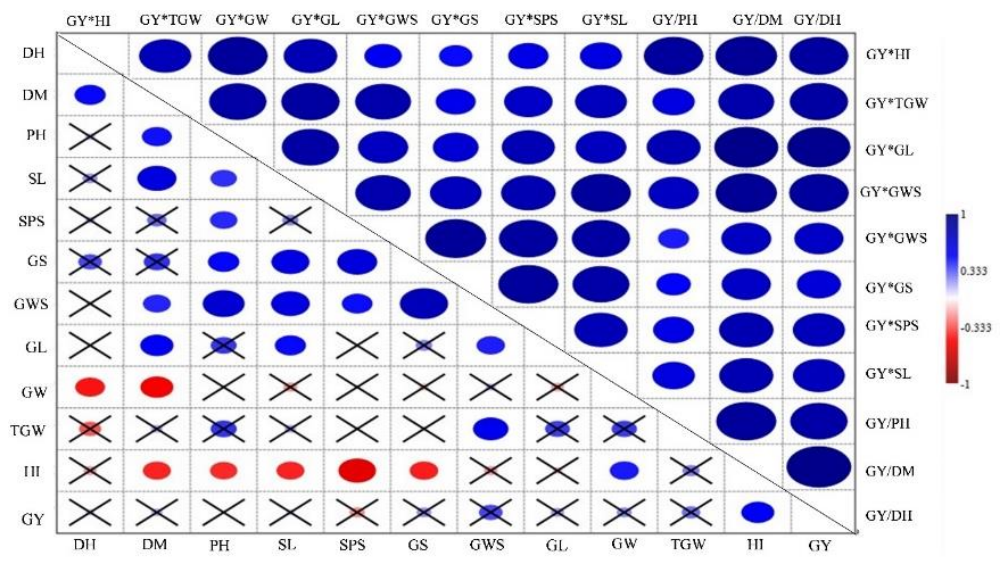

Fig. 2: Correlation among traits (lower diagonal) and grain yield trait combinations (upper diagonal) of 24 wheat genotypes The cross symbol represents the non-significance association

(Fig. 4). The GYT biplot showed that the polygon consisted of eight genotypes which were present at the farthest distances from the origin at vertex positions to encompass all the remaining genotypes. These vertex genotypes included DF1906, DF1912, DF1917, DF1923, DF1916, DF1914, DF1909 and DF1904. Additionally, the eight perpendicular rays radiating from the origin of polygon distributed the biplot into eight sectors, among which only three sectors possessed the yield trait combinations. The first sector between $1^{\text {st }}$ and $8^{\text {th }}$ radiating rays comprised of four yield trait combinations viz., GY*SL, GY*SPS, $\mathrm{GY} * \mathrm{GW}$ and $\mathrm{GY} * \mathrm{GS}$ and 6 advanced lines viz., DF1906,
DF1907, DF1905, DF1910, DF1908 and DF1903 among which DF1906 was the winner genotype. It implied that the genotype DF1906 had the best trait profile for these yield components as compared to rest of the genotypes. The second sector between $1^{\text {st }}$ and $2^{\text {nd }}$ radiating rays harbored six yield trait combinations including GY/DH, GY/DM, GY*GW, GY*TGW, and GY*GL with only one vertex genotype (DF1912) while third sector between $2^{\text {nd }}$ and $3^{\text {rd }}$ rays also had only one genotype (DF1917) present at vertex position which performed quite well for GY/PH and GY*HI. Taken together these results depict that the advanced line DF1912 and DF1917 were early maturing 


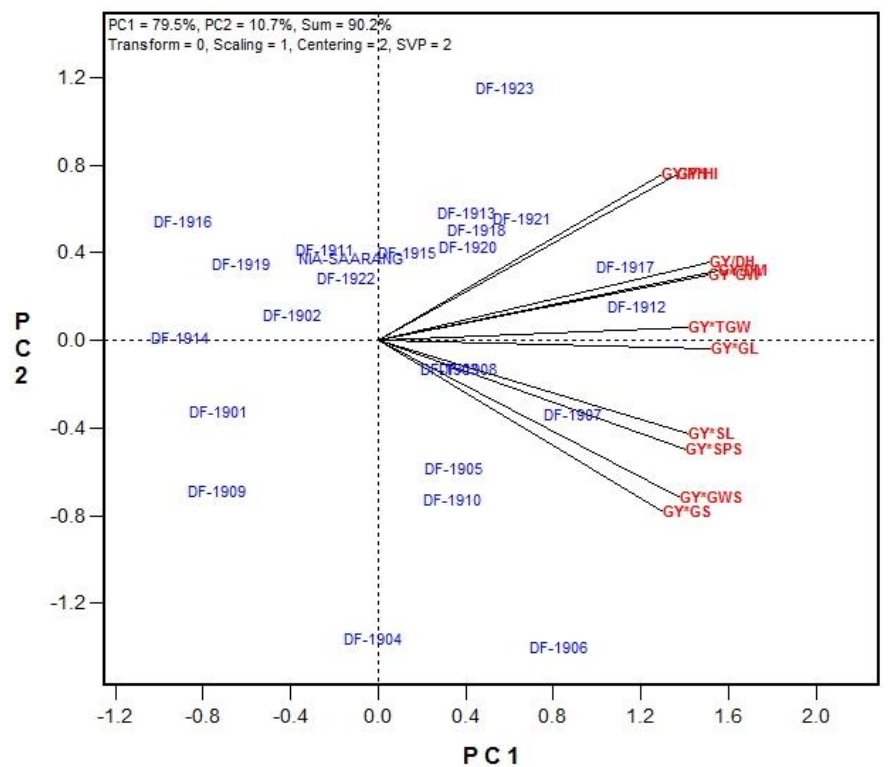

Fig. 3: The test vector view of genotype by yield*trait (GYT) biplot to represent the association among grain yield trait combinations

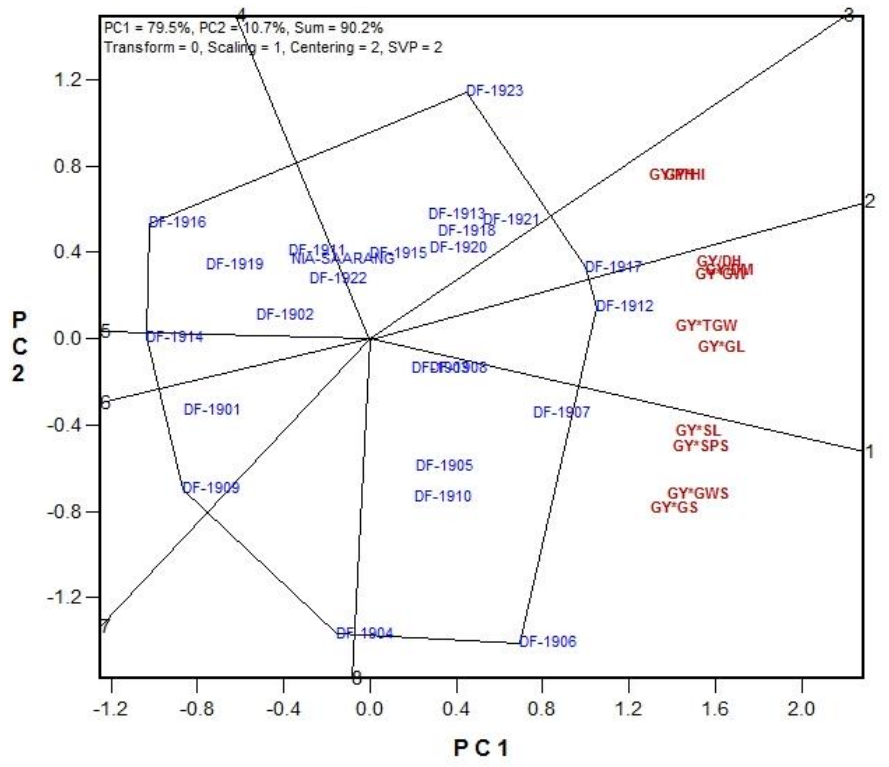

Fig. 4: The best view of genotype by yield*trait (GYT) biplot to represent the vertex genotypes of the polygon

short stature genotypes with excellent trait profile for grain weight, grain yield and harvest index. In contrast, the polygon view also described that the remaining 16 genotypes including check cultivar were present in those sectors which had any yield trait combinations, implying that these genotypes were the poor performer of studied traits in combination to grain yield as compared to rest of genotypes (Fig. 4).

The ATC view of GYT biplot presented in Fig. 5, best explained the ranks of 24 genotypes based on the performance of multiple traits and usefulness. Overall, the ATC biplot plotted 13 genotypes at the right side of the double head arrow on the ATA axis to represent as better performers than the average of all the yield trait combinations. Hence, these genotypes could be ranked as DF1912 > DF1917 > DF1907 > DF1906 > DF1921> DF1923 > DF1918>DF1920> DF1913> DF1908> DF1905 > DF1910 > DF1903 (Fig. 5). The positive values of mean superior index (SI) of all these genotypes also authenticated the outcomes of GYT biplot (Table 1). The advanced line DF1915 present at the origin of biplot had the same level of performance as that of overall average of all the traits. In contrast, 10 genotypes including check cultivar NIA-Saarang appended on the left side of double head 
Faheem et al. / Intl J Agric Biol, Vol 25, No 6, 2021

Table 1: The mean standardized genotype by yield*trait data of two years and the mean superiority index (SI) of 24 wheat genotypes

\begin{tabular}{|c|c|c|c|c|c|c|c|c|c|c|c|c|}
\hline Genotypes & GY/DH & GY/DM & GY/PH & $\mathrm{GY} * \mathrm{SL}$ & GY*SPS & GY*GS & GY*GWS & $\mathrm{GY} * \mathrm{GL}$ & $\mathrm{GY}^{*} \mathrm{GW}$ & GY*TGW & GY*HI & Mean SI \\
\hline DF-1901 & $\begin{array}{l}-1.69 \\
\end{array}$ & -1.25 & $\begin{array}{l}-1.07 \\
\end{array}$ & $\begin{array}{l}-0.87 \\
\end{array}$ & -0.92 & -0.65 & -1.28 & $\begin{array}{l}-0.85 \\
\end{array}$ & -1.87 & -1.67 & -1.39 & -1.23 \\
\hline DF-1902 & -0.61 & -0.89 & -0.68 & -0.75 & -0.69 & -0.81 & -0.99 & -0.49 & -0.69 & -0.91 & -0.78 & -0.75 \\
\hline DF-1903 & 0.44 & 0.11 & -0.33 & -0.03 & 0.52 & -0.39 & 0.47 & 0.67 & 0.49 & 1.28 & -0.35 & 0.26 \\
\hline DF-1904 & -0.82 & -0.63 & -0.98 & 0.93 & 0.03 & 1.13 & 0.87 & -0.59 & -0.50 & -0.55 & -1.18 & -0.21 \\
\hline DF-1905 & 0.19 & 0.20 & -0.17 & 0.89 & 0.53 & 0.66 & 0.60 & 0.52 & -0.12 & 0.26 & -0.20 & 0.30 \\
\hline DF-1906 & 0.62 & 0.79 & 0.06 & 0.67 & 2.21 & 2.53 & 2.02 & 1.12 & 0.71 & 0.40 & -0.10 & 1.00 \\
\hline DF-1907 & 0.70 & 0.82 & 0.62 & 1.78 & 1.16 & 0.82 & 1.31 & 1.32 & 1.04 & 1.55 & 0.86 & 1.09 \\
\hline DF-1908 & 0.79 & 0.56 & -0.07 & 0.72 & 0.58 & 0.13 & 0.30 & 0.25 & 0.56 & 0.56 & -0.13 & 0.39 \\
\hline DF-1909 & -1.40 & -1.59 & -1.88 & -1.14 & -0.93 & -0.43 & -0.61 & -1.15 & -1.54 & -1.39 & -1.52 & -1.24 \\
\hline DF-1910 & 0.17 & -0.22 & -0.60 & 0.77 & 0.23 & 0.32 & 1.29 & 0.36 & -0.11 & 1.24 & -0.20 & 0.30 \\
\hline DF-1911 & -0.27 & -0.53 & 0.47 & -0.25 & -0.36 & -0.57 & -1.28 & -0.49 & -0.74 & -1.36 & -0.46 & -0.53 \\
\hline DF-1912 & 1.76 & 1.66 & 1.50 & 1.70 & 1.17 & 1.43 & 1.18 & 1.67 & 2.04 & 1.04 & 1.31 & 1.50 \\
\hline DF-1913 & 1.07 & 0.68 & 1.27 & -0.03 & 0.17 & 0.00 & 0.09 & 0.12 & -0.02 & 0.56 & 0.39 & 0.39 \\
\hline DF-1914 & -1.86 & -1.63 & -1.29 & -1.96 & -1.06 & -1.34 & -1.23 & -1.95 & -1.39 & -1.21 & -1.27 & -1.47 \\
\hline DF-1915 & 0.22 & -0.08 & -0.10 & -0.29 & -0.04 & -0.58 & -0.12 & -0.04 & -0.07 & 0.42 & 0.70 & 0.00 \\
\hline DF-1916 & -1.33 & -1.54 & -1.21 & -1.40 & -2.60 & -1.79 & -1.47 & -1.57 & -1.20 & -1.02 & -1.00 & -1.47 \\
\hline DF-1917 & 1.10 & 1.73 & 1.69 & 1.43 & 1.05 & 1.36 & 1.14 & 1.71 & 1.35 & 1.18 & 2.06 & 1.44 \\
\hline DF-1918 & 0.61 & 0.74 & 0.86 & 0.15 & -0.08 & 0.38 & 0.21 & 0.27 & 0.61 & 0.34 & 0.95 & 0.46 \\
\hline DF-1919 & -1.27 & -1.03 & -0.03 & -0.97 & -0.95 & -1.09 & -1.52 & -1.26 & -1.06 & -1.57 & -1.10 & -1.08 \\
\hline DF-1920 & 0.57 & 0.63 & 0.77 & -0.40 & 1.10 & 0.34 & 0.05 & -0.58 & 1.12 & 0.02 & 0.80 & 0.40 \\
\hline DF-1921 & 0.81 & 1.20 & 1.31 & 0.75 & 0.30 & 0.54 & 0.10 & 1.02 & 0.70 & 0.43 & 1.09 & 0.75 \\
\hline DF-1922 & -0.61 & -0.36 & -0.42 & -0.59 & -0.59 & -0.74 & -0.52 & -0.10 & -0.38 & -0.08 & -0.02 & -0.40 \\
\hline DF-1923 & 1.07 & 0.91 & 1.30 & -0.07 & -0.10 & -0.45 & 0.08 & 0.80 & 0.98 & 0.87 & 1.54 & 0.63 \\
\hline NIA-Saarang & -0.25 & -0.27 & -1.02 & -1.03 & -0.72 & -0.82 & -0.70 & -0.75 & 0.11 & -0.40 & -0.03 & -0.53 \\
\hline
\end{tabular}

GY, grain yield; DH, days to heading; DM, days to physiological maturity; PH, plant height; SL, spike length; SPS; number of spikelets per spike; GS, number of grains per spike; GWS, grain weight per spike; GW, grain width; GL, grain length; TGW, 1000-grain weight; HI, harvest index

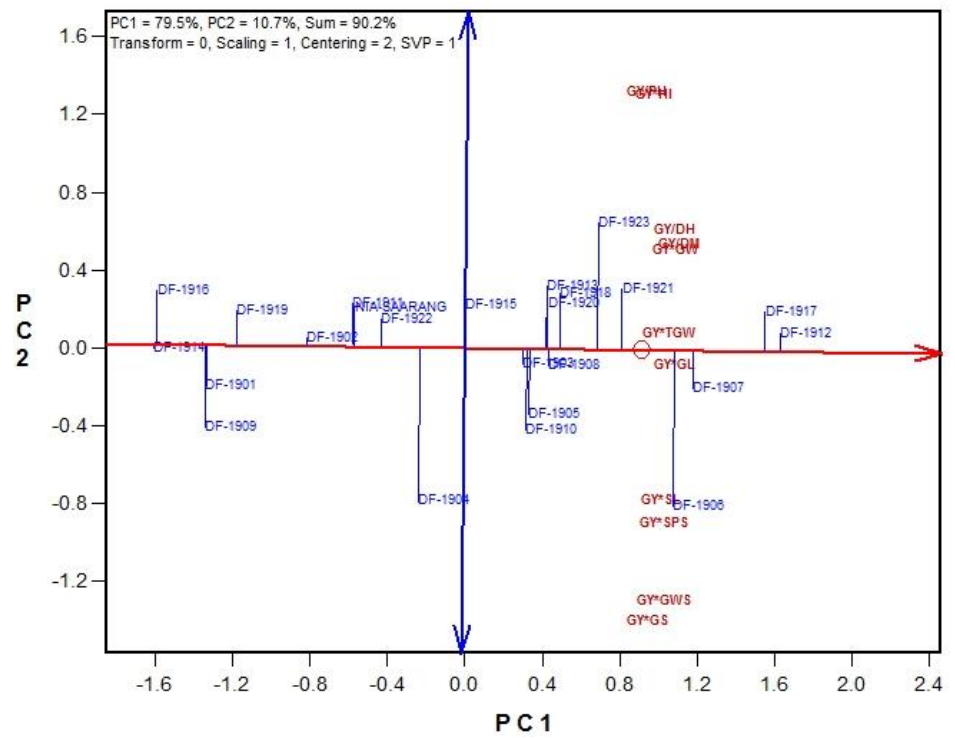

Fig. 5: The average tester coordination (ATC) view of genotype by yield*trait (GYT) biplot to rank wheat genotypes based on multiple traits

arrow had the poorer performance than the average of all the traits. All these genotypes had negative mean SI values (Table 1), which implied that these genotypes had not the suitable combinations of traits as desired. It is evident from the ATC biplot that the four advanced line DF1912, DF1917, DF1907 and DF1908 had the trait profiles better than the overall average of all the traits, hence, outclassed other contesting genotypes including check cultivar. Among these lines, DF1912 was at the top position followed by DF1917 which had the potential to produced maximum grain yield at the expense of studied traits and signified ideotypes of the testing panel of genotypes to be selected on the basis of breeding objectives. Both of these advanced lines DF1912 and DF1917 had the maximum mean SI values of 1.50 and 1.44 , respectively for which each trait contributed positively toward grain yield (Table 1).

\section{Discussion}

During the development of crop cultivars, plant breeders 
usually focus on the selection of a couple of specific traits in connection with main economical trait. It is because selection based on multiple traits may disturb the balance among the traits due to negative association among different traits and the interaction of these traits with the environment (Yan et al. 2007; Kendal 2019). In contrast, the value of cultivar increases for end users when it is evolved on the basis of multiple traits (Karahan and Akgun 2020). Therefore, statisticians and breeders are continuously putting effort to develop an effective model to select superior genotypes based on multiple traits. In this regard, initially genotype by trait (GT) analysis was proposed to understand the relationship among traits and genotypes (Yan and Rajcan 2002) and was utilized by many breeders in cereals as well as in other crops (Rubio et al. 2004; Oladejo et al. 2011; Legesse et al. 2013; Paramesh et al. 2016). However, this GT biplot model cannot show the strengths and weaknesses of a genotype, hence deprives of providing a decisive power of selection or rejection of given genotype (Yan et al. 2019). An extension of GT biplot analysis named as genotype by yield*trait (GYT) biplot model was recently proposed by Yan and Fregeau-Reid (2018) to select or reject the genotypes based on multiple traits (Merrick et al. 2020). The same model following all the steps and principles was applied in the present study to select the best advanced lines based on multiple traits from the panel of 24 contesting wheat genotypes. At first, GT biplot was constructed from the average standardized data set of two years revealed that the GY was strongly linked with TGW and weak correlation was found for GW, whereas no other traits had the significant association with grain yield. Additionally, other yield components like GWS, GS SPS, SL and GL were associated with each other in different strengths of associations while these traits were negatively correlated with harvest index and GW (Fig. 1). The Pearson correlation table also validated the same trend of associations among traits with some exceptions (Fig. 2). According to correlation table, the grain yield was significantly associated with only HI not with TGW. This might be due to the fact that not enough variation was found among the genotypes for grain yield as also revealed by short vector length of GY in GT biplot. The other reason included the fitness of good of GT biplot which was quite low as compared to GYT biplot $(90.2 \%)$ which means that GT biplot could only explain $57 \%$ of the total variation.

At second step of GYT biplot analysis, the GT table was transformed to GYT biplot by multiplying or dividing grain yield with other traits depending upon the breeding objectives. As, we were looking for early maturing, short stature and high yielding genotypes for the agro-climatic conditions of Pakistan, therefore, the grain yield was divided by days to heading, days to physiological maturity and plant height while all other trait parameters were multiplied to get the standardized GYT table (Table 1). Three unique views of GYT biplot were constructed (Fig. 3, 4 and 5) from this data set which explained $90.2 \%$ of the total data variation, significantly much higher than GT biplot. Like other studies, our results also demonstrated that GYT biplot was better and accurate than the GT biplot to display the actual variation in dataset for reliable selection (Yan et al. 2019). The tester vector view of GYT biplot demonstrated that almost all the yield trait combinations were associated with each other at different degree of association which could not be explained by GT biplot. Secondly, the genotypes present within the acute angles of tester vectors (yield trait combinations) had the trait profile contributed positively towards grain yield which could not be assessed by GT biplot. For instance, GT biplot depicted that the advanced lines DF1909, DF1904 and DF1905 had the features of early maturing (Fig. 1) but the GYT biplot placed these genotypes away from all the tester vectors related to early maturity (GY/DH and GY/DM) advocating that these genotypes may had the early maturing featuring but not contributed positively towards grain yield which was the goal of any breeding program. A similar case was with DF1923 which had the highest HI but the strength of this trait was not in the optimum balance of other traits to be selected as an ideotype. However, all these genotypes could be used as donor parents for the improvement of these traits in breeding programs. Recently, Yan et al. (2019) also pointed out that the economic value of any trait in a cultivar depends upon the level of main target trait i.e., grain yield.

The polygon views also known as which is best for what biplot is an excellent graphical presentation of trait profile of genotypes. The results of polygon view showed that 8 genotypes were the polygon vertex among which the advanced line DF1906 was the best genotype for main yield components including spike length, number of spikelets per spike, grain weight per spike and number of grains per spike, while DF1912 was early maturing coupled with highest thousand grain weight and DF1917, was low stature with highest harvest index (Fig. 4). In contrast, the remaining five vertex genotypes DF1923, DF1916, DF1914, DF1909 and DF1904 were winners of respective sectors but did not possess the desired level of multiple traits to be selected as ideotype. The ATC view is the most unique feature of GYT biplot as it displays the ranks of contesting genotypes based on strengths and weaknesses of each genotypes which cannot be viewed in other biplots including GT biplot (Karahan and Akgun 2020; Yan et al. 2019). This view categorized the inferior and superior genotypes groups by drawing a perpendicular doubled head arrow on ATA axis which were present on left and right side of the arrow, respectively. Additionally, a small hollow circle on main ATA axis representing the average of all yield trait combinations further subdivided the superior group into the most desirable genotypes (ideotype) and desirable ones (Yan and Fregeau-Reid 2018). In this study, the ATC biplot grouped 13 genotypes as superior and 9 genotypes as inferior while one genotype DF1915 was at the boundary of these groups (Fig. 5). All the superior advanced lines outclassed the check $c v$. NIA-Saarang while four 
genotypes crossed the small hollow circle on ATA axis. Among these genotypes, DF-1912 and DF1917 had the best traits profile as also depicted by GYT table that all the yield combinations of these genotypes had positive values. Between these two ideotypes, DF1912 was better and more stable than the DF1917 as it had the shorter projection on ATA axis. In contrast, the nine inferior genotypes had the poor trait profile when assessed in combination with yield, hence could be rejected on the basis of multiple traits.

\section{Conclusion}

This research endeavor was meant to demonstrate the application of GYT biplot approach to select the superior wheat advance line based on multiple traits to release the high value cultivars for end users. The GYT biplot analysis clearly categorized the contesting wheat lines into superior and inferior group and recommended two ideotypes i.e., DF1912 and DF1917 on the basis of balanced trait profile. Our findings also strengthen the argument that the GYT biplot analysis is better than other selection indices and guaranteed the selection of superior genotypes and rejection of inferior ones when evaluated on multiple traits in connection with yield.

\section{Acknowledgements}

The research project was financially supported by the funds of Pakistan Atomic Energy Commission.

\section{Author Contributions}

MF developed the experimental material, designed and executed the experiment, recorded the data, and wrote the manuscript; KAL and SMA developed the experimental material, MK statistically analyzes the data, MAS supervised and reviewed the manuscript.

\section{Conflict of Interest}

Authors declare no conflict of interest.

\section{Data Availability}

The data will be made available on fair request to the corresponding author.

\section{Ethics Approval}

Not applicable.

\section{References}

Bernardo R (2010). Selection for multiple trait. In: Breeding for Quantitative Traits in Plants, pp:343-358. Stemma press, Minnesota, USA

Bos I, P Caligari (2007). Index selection and independent culling method. In: Selection Methods in Plant Breeding, $2^{\text {nd }} e d n, ~ p p: 318-324$. Springer, Netherlands

Boureima S, Y Abdoua (2019). Genotype by yield* trait combination biplot approach to evaluate sesame genotypes on multiple traits basis. Turk J Field Crops 24:237-244

Godshalk E, D Timothy, J Burns (1988). Effectiveness of index selection for switchgrass forage yield and quality. Crop Sci 28:825-830

Joshi KD, AU Rehman, G Ullah, MF Nazir, M Zahara, J Akhtar, M Khan, A Baloch, J Khokhar E Ellahi, A Khan, M Suleman, M Imtiaz (2017). Acceptance and competitiveness of new improved wheat varieties by smallholder farmers. J Crop Improv 31:608-627

Karahan T, I Akgün (2020). Selection of barley (Hordeum vulgare) genotypes by GYT (genotype $\times$ yield $\times$ trait) biplot technique and its comparison with GT (genotype $\times$ trait). Appl Ecol Environ Res 18:1347-1359

Kendal E (2019). Comparing durum wheat cultivars by genotype $x$ yield $\times$ trait and genotypex trait biplot method. Chil J Agric Res 79:512-522

Legesse H, N Dechassa, S Gebeyehu, G Bultosa, F Mekbib (2013). Multivariate analysis as a tool for indirect selection of common bean genotypes (Phaseolus vulgaris L.) for soil acidity tolerance under field conditions. Sci Technol Arts Res J 2:07-15

Li J, I Dundas, C Dong, G Li, R Trethowan, Z Yang, S Hoxha, P Zhang (2020). Identification and characterization of a new stripe rust resistance gene $Y r 83$ on rye chromosome 6R in wheat. Theor Appl Genet 133:1095-1107

Merrick LF, KD Glover, D Yabwalo, E Byamukama (2020). Use of genotype by yield* trait (GYT) analysis to select hard red spring wheat with elevated performance for agronomic and disease resistance traits. Crop Breed Gene Genomics 2; Article e200009

Mohammadi R (2019). Genotype by yield*trait biplot for genotype evaluation and trait profiles in durum wheat. Cer Res Commun 47:541-551

Oladejo AS, R Akinwale, I Obisesan (2011). Interrelationships between grain yield and other physiological traits of cowpea cultivars. Afr Crop Sci J 19:189-200

Paramesh M, D Reddy, MS Priya, P Sumathi, P Sudhakar, K Reddy (2016). GT biplot analysis for yield and drought related traits in mung bean (Vigna radiata Wilczek). Electr J Plant Breed 7:538-543

Rubio J, J Cubero, L Martin, M Suso, F Flores (2004). Biplot analysis of trait relations of white lupin in Spain. Euphytica 135:217-224

Simmonds NW, J Smartt (1999). Genetic aspects: Populations and selection. In: Principles of Crop Improvement, $2^{\text {nd }}$ edn, pp:62-114. Oxford, Blackwell, USA

Tilman D, C Balzer, J Hill, BL Befort (2011). Global food demand and the sustainable intensification of agriculture. Proc Natl Acad Sci 108:20260-20264

Yan W, I Rajcan (2002). Biplot analysis of test sites and trait relations of soybean in Ontario. Crop Sci 42:11-20

Yan W, J Fregeau-Reid (2018). Genotype by yield* trait (GYT) biplot: A novel approach for genotype selection based on multiple traits. Sci Rep 8; Article 8242

Yan W, J Fregeau-Reid, N Mountain, J Kobler (2019). Genotype and management evaluation based on genotype by yield*trait (GYT) analysis. Crop Breed Genet Genomics 1; Article e190002

Yan W, MS Kang, B Ma, S Woods, PL Cornelius (2007). GGE biplot $v s$. AMMI analysis of genotype-by-environment data. Crop Sci 47:643-653 